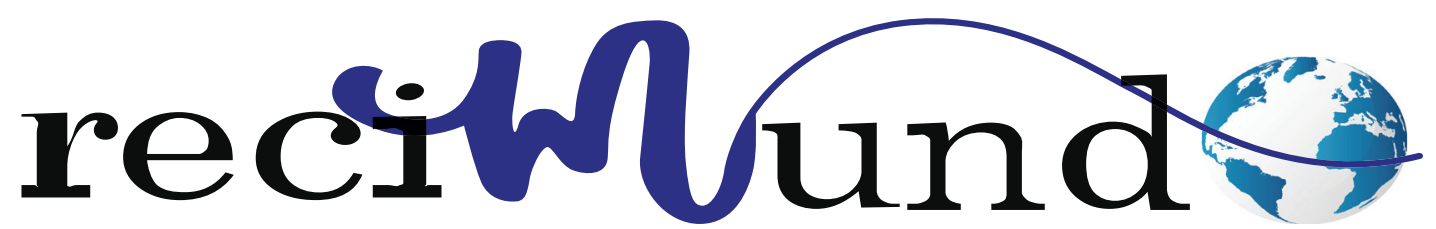

Revista Científica Mundo de la Investigación y el Conocimiento

DOI: 10.26820/recimundo/5.(2).abril.2021.128-139

URL: https://recimundo.com/index.php/es/article/view/1047

EDITORIAL: Saberes del Conocimiento

REVISTA: RECIMUNDO

ISSN: 2588-073X

TIPO DE INVESTIGACIÓN: Artículo de revisión

Código UNESCO: 32 Ciencias Médicas

PAGINAS: $128-139$

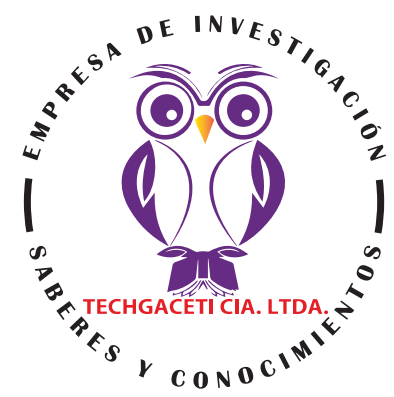

\title{
Manejo integral de la insuficiencia renal aguda
}

Integral management of acute renal failure

Gerenciamento abrangente da insuficiência renal aguda

Tatiana Jeannette Arguello Molina'; Carlos Javier Quirós Rumbea2; Kattia Liseth Daza Bermeo3;

Francisco David Macías Vélez ${ }^{4}$

RECIBIDO: 15/01/2021 ACEPTADO: 20/03/2021 PUBLICADO: 01/04/2021

1. Magister en Gerencia Hospitalaria; Médico; Investigador Independiente; Guayaquil, Ecuador; tatedanvican1985@gmail. com; (iD https://orcid.org/0000-0003-3465-606X

2. Médico de la Universidad Particular de Especialidades Espíritu Santo; Investigador Independiente; Guayaquil, Ecuador; cquiros8@hotmail.com; iD https://orcid.org/0000-0002-8179-1320

3. Médico Cirujano de la Universidad Laica Eloy Alfaro de Manabí; Guayaquil, Ecuador; kattiadazza90@gmail.com; (iD https://orcid.org/0000-0003-2842-2419

4. Médico Cirujano de la Universidad Laica Eloy Alfaro de Manabí; Guayaquil, Ecuador; bekraujis.vyras@gmail.com; (iD https://orcid.org/0000-0002-6876-9575

\section{CORRESPONDENCIA}

Tatiana Jeannette Arguello Molina

tatedanvican1985@gmail.com

Guayaquil, Ecuador

() RECIMUNDO; Editorial Saberes del Conocimiento, 2021 


\section{RESUMEN}

Las enfermedades de insuficiencia renal actualmente se consideran como una patología compleja que en sus inicios puede ser tratada de manera fácil y sencilla. Sin embargo, si la enfermedad no es abordada a tiempo, puede desencadenar hacia la insuficiencia renal terminal generando complicaciones que deben ser tratadas a través del tratamiento con diálisis y renal sustitutivo. La evolución del paciente hacia la perdida de la función renal se ocasiona de manera progresiva, producto de causas asociadas con la hipertensión arterial (HTA) y la diabetes mellitus. Esta afección, se considera común y se encuentra con frecuencia tanto en la práctica comunitaria como en los pacientes hospitalizados. Tiene gran impacto en aquellos pacientes que llegan a ser sometidos a diálisis debido a los cambios de vida a los cuales son sometidos y la aceptación del tratamiento, lo que reduce sus expectativas de vida. No obstante, sigue siendo una enfermedad heteróloga, y seguir los principios básicos facilita la investigación donde el tratamiento inicial sigue una vía estándar en la mayoría de los pacientes. Este artículo se basa en mostrar y brindar información acerca de las estrategias terapéuticas, incluidas aquellas en situaciones especiales, en aras de ayudar al médico a decidir cuándo derivar a un nefrólogo y cuándo considerar la terapia de reemplazo renal.

Palabras clave: Diálisis, insuficiencia renal, diálisis, calidad de vida.

\section{ABSTRACT}

The injuries of renal insufficiency are currently considered to be a complex pathology that can be treated in a common and common way. Without the embargo, if not affected by the time, it is possible to undermine the renal terminal insufficiency generating complications that must be treated through treatment with dual and renal replacement. The evolution of patients has lost renal function by progressive manners, the product of cases associated with arterial hypertension (HTA) and diabetes mellitus. This action shall be taken into account and provided with the frequency of Community practice in hospitalized patients. There is a great deal of impact on patients who are expected to receive legal changes to life changes to their needs and acceptance of treatment, which reduces their expectations of life. However, a heterologous nurse should be provided and basic principles facilitated by the investigation of the initial treatment should be established in the patients' history. This article is based on the provision and provision of information on therapeutic strategies, including in special situations, in the case of medical aids, and how to derive a nephrologist and need to consider renal relapse therapy.

Keywords: Dialisis, renal insufficiency, dialisis, quality of life.

\section{RESUMO}

As lesões por insuficiência renal são atualmente consideradas como uma patologia complexa que pode ser tratada de uma forma comum e comum. Sem o embargo, se não for afetado pelo tempo, é possível minar a insuficiência renal terminal gerando complicações que devem ser tratadas através de tratamento com substituição dupla e renal. A evolução dos pacientes perdeu a função renal através de modos progressivos, produto de casos associados à hipertensão arterial (HTA) e diabetes mellitus. Esta ação deve ser levada em conta e proporcionada com a freqüência da prática comunitária em pacientes hospitalizados. Há um grande impacto sobre os pacientes que se espera que recebam mudanças legais nas mudanças de vida para suas necessidades e aceitação de tratamento, o que reduz suas expectativas de vida. Entretanto, uma enfermeira heteróloga deve ser providenciada e princípios básicos facilitados pela investigação do tratamento inicial devem ser estabelecidos na história dos pacientes. Este artigo se baseia no fornecimento e na prestação de informações sobre estratégias terapêuticas, inclusive em situações especiais, no caso de auxílios médicos, e como derivar um nefrologista e a necessidade de considerar a terapia da recidiva renal.

Palavras-chave: Dialisisis, insuficiência renal, diálise, qualidade de vida. 


\section{Introducción}

La insuficiencia renal aguda (IRA) "es una reducción potencialmente reversible de la capacidad del riñón para excretar desechos nitrogenados y mantener la homeostasis de líquidos y electrolitos, que suele ocurrir durante horas o días" (Glynne \& Allen, 2014). El enfoque clínico inicial es idéntico en todos los pacientes: una anamnesis y un examen minuciosos con tratamiento simultáneo de cualquier característica potencialmente mortal (por ejemplo, hiperpotasemia grave). El tratamiento posterior debe centrarse en determinar la causa, que puede exigir un tratamiento específico, mantener el estado de volumen del paciente y evitar más agresiones nefrotóxicas.

Los patrones de morbilidad y mortalidad por enfermedad en todo el mundo han cambiado, tanto en el mundo desarrollado como en el emergente. En todo el siglo 20, las enfermedades infecciosas fueron la principal causa de muerte y de discapacidad. No obstante, en este siglo, a pesar de que las enfermedades infecciosas siguen marcando un número significativo de mortalidad, las enfermedades no infecciosas se han convertido también, en una de las principales causas de mortalidad y morbilidad en todo el mundo. Esto se debe al tipo de enfermedades que causan insuficiencia renal crónica y en su presentación y progresión.

Las enfermedades de deficiencia renal, se consideran una alteración progresiva, con una velocidad de deterioro que varía de acuerdo a la etiología tanto del paciente como de la enfermedad renal como tal. El incremento en la morbilidad que se ha registrado en los hospitales, se debe a la evaluación tardía de los nefrólogos debido a que los pacientes son remitidos al especialista ya en estado avanzado probablemente por los costos que esto genera, lo que incrementa los casos de morbilidad y mortalidad en pacientes con insuficiencia renal. En tal sentido, se puede indicar que" la iden- tificación precoz de los pacientes con dicha afección permite realizar tratamientos que limitan la progresión del daño renal y modificar los factores de riesgo asociados que contribuyen al aumento de la morbilidad en los afectados" (Firth, 2008). En esta labor de detección juegan un papel fundamental, los equipos de atención primaria, dado que, en sus estadios iniciales, habitualmente son asintomática; su identificación suele tener lugar de forma accidental o en análisis solicitados a pacientes de riesgo (hipertenso o diabético).

El desarrollo de este artículo busca construir un registro bibliográfico de investigaciones previamente realizadas por especialistas, con el objetivo de brindar fundamentos importantes para el estudio, por lo cual cada análisis de los temas seleccionados para la redacción del campo investigativo hace posible el desarrollo y redacción que conforman el tema seleccionado.

\section{Metodología}

El camino metodológico que caracteriza al presente artículo, se encuentra determinado por la presencia de argumentos de investigación caracterizados por ofrecer informaciones pertinentes al tema debidamente seleccionado, el mismo se apoya en las ideas dadas por lo citado por Ortiz y García (2018) para lograr eficiencia en una investigación es importante seleccionar el método que se convierte en una herramienta de recopilación de información determinada por momentos teóricos y prácticos". (p. 65)

En consecuencia, para dar continuidad al proceso investigativo se hace necesario identificar el método analítico como pieza fundamental que gira las acciones básicas encargadas de encaminar el hecho científico bajo una valoración precisa y coherente de los aspectos que identifican previamente el fenómeno en estudio. Tal como lo define Ortiz y García (ob.cit) es aquel método que 
"consiste en la desmembración de un todo descomponiéndolo en sus elementos para observar las causas, naturaleza y efectos" (p.65). De allí, que su introducción en este artículo, hace posible desglosar el contenido temático en partes esenciales que al sumarse logran construir básicamente las apreciaciones generales que el autor considera pertinente.

\section{Tipo de Investigación}

Una vez seleccionado el método se hace necesario recurrir a la ubicación dentro del campo científico al artículo, lo que asegura su relación con los estudios documentales visto por Zambrano (2018) "como la recopilación de informaciones provenientes de materiales impresos, audiovisuales, tecnológicos entre otras, para expresar nuevas ideas acerca de un tema en particular". (p.33). Es decir, mediante este tipo de investigación, se puede replantear nuevas valoraciones referidas a un contenido en particular, que hace posible conjugar diferentes interpretaciones para finalmente complementar sus beneficios dentro de una teoría en particular.

\section{Fuentes Documentales}

La conducción de la dinámica científica amerita para ser viable a un proceso veraz, el complemento de aquellas fuentes documentales que le dan oportunidad de encontrar de forma precisa el respectivo dominio de un fenómeno de acuerdo con sus características especiales que le asignan un conocimiento enmarcado en una realidad previa. En tal sentido Zambrano (ob.cit) destaca que las fuentes documentales "es la suma de técnicas que permiten orientar el trabajo desde una perspectiva cotidiana para cumplir con los diferentes procedimientos mediante explicaciones".(p.35). Por lo tanto, su aplicación en el estudio, permite asegurar con antelación, la revisión de diferentes documentos propios y relacionados con el tema, para luego elaborar su contenido que quedará como representación del fenómeno en estudio.

\section{Técnicas para la Recolección de la Infor- mación}

Con el fin de darle operatividad a la investigación, se busca primeramente plantear la definición de técnica dada por Zambrano (ob.cit) "son los diferentes procedimientos para el aprovechamiento científico de los elementos de la naturaleza y sus derivados". (p.35). Según lo citado, permite interpretar que darle la respectiva complejidad al análisis que se busca del tema previo, se hace necesario adecuar las apreciaciones en función a los procedimientos que den cabida a un mayor aprovechamiento de los elementos presente en la naturaleza del tema seleccionado.

De este modo, durante la fase de recolección de la información, se llevaron a cabo acciones especiales que dieron cabida a incorporar las técnicas de fuentes de información definidas por Zambrano (ob.cit) "como aquellas herramientas básicas para las investigaciones documentales que conllevan al uso óptimo y racional de los recursos documentales" (p.35). En consecuencia mediante su utilización, se puede organizar los diferentes contenidos disponibles en la web considerando que aquella herencia de la globalización permite acceder a mayor y mejor información a través de las herramientas tecnológicas. El motor de búsqueda ha sido herramientas académicas de la web que direccionan específicamente a archivos con validez y reconocimiento científico, descartando toda información no confirmada o sin las respectivas referencias bibliográficas.

\section{Resultados}

\section{Causas de insuficiencia renal aguda}

De acuerdo con Glynne \& Allen, (2014) "clásicamente, las causas de IRA se han subdi- 
vidido en tres grupos: prerrenal, intrínseca y postrenal". Si bien existe una superposición considerable entre estos, especialmente los dos primeros, sigue siendo una guía clínica útil.

\section{Prerrenal}

Perfusión renal reducida, atribuible a hipovolemia, hipotensión o fármacos, que es reversible al corregir la causa subyacente.

\section{Intrínseco}

Comúnmente resulta de lesión de las células tubulares o muerte atribuible a insuficiencia prerrenal prolongada o inadecuadamente corregida: necrosis tubular aguda isquémica (NTA).

Los fármacos también pueden causar daño tubular directo: NTA nefrotóxica. La nefritis intersticial aguda (NIA) puede resultar de reacciones alérgicas a medicamentos, infecciones u ocasionalmente enfermedad sistémica (por ejemplo, sarcoidosis). La inflamación glomerular fulminante, glomerulonefritis rápidamente progresiva (por ejemplo, debido a vasculitis), es la menos común, pero exige la investigación más urgente (Kim \& Han, 2012).

\section{Posrrenal}

La obstrucción de la salida de orina de ambos riñones, o de un solo riñón en funcionamiento, puede surgir en cualquier lugar desde la pelvis renal hasta la uretra. El alivio de la obstrucción generalmente conduce a la recuperación de la función.

La insuficiencia prerrenal "es la causa más frecuente, al menos en los pacientes hospitalizados, aunque la obstrucción secundaria a enfermedad prostática es tan común en algunos estudios comunitarios" (Feest, Round, \& Hamad, 2003). La enfermedad intrínseca "es muy probablemente atribuible a NTA isquémica (50\% de los casos de
IRA intrínseca) con NTA nefrotóxica, nefritis intersticial y glomerulonefritis que representan el 35\%, 10\% y 5\% de los casos, respectivamente" (Thadhani, Pascual, \& Bonventre, 2006).

La afección es a menudo multifactorial, por ejemplo, el paciente séptico e hipotenso que recibe aminoglucósidos y contraste intravenoso. Los pacientes de edad avanzada, los pacientes diabéticos y aquellos con enfermedad renal preexistente tienen un mayor riesgo.

\section{Enfoque clínico del paciente}

Los autores Brezis \& Rosen, (2015) exponen que "los fundamentos para el abordaje del paciente con IRA son los siguiente":

1. Trate cualquier característica potencialmente mortal.

- La hipotensión, el shock y la insuficiencia respiratoria deben ser evidentes de inmediato al evaluar al paciente, y claramente estos exigen un tratamiento urgente. Es menos probable que la hiperpotasemia sea inmediatamente obvia. A menos que los cambios sean evidentes en el ECG o en la monitorización cardíaca, solo se harán evidentes cuando la química esté disponible.

2. Identificar cualquier causa de IRA que justifique un tratamiento específico.

Muchos pacientes con IRA presentan otros diagnósticos. La deshidratación secundaria a pérdidas gastrointestinales, neumonía, obstrucción intestinal y nuevo deterioro de la capacidad funcional en el paciente anciano son a menudo los diagnósticos iniciales al tomar un diagnóstico de insuficiencia renal solo se realiza cuando los parámetros de laboratorio están disponibles más adelante. A continuación, el médico debe plantearse las siguientes preguntas (véase el cuadro $\mathrm{N}^{\circ} 1$ ). 
Tabla 1. Historia y expoloración en la insuficiencia renal aguda

HISTORIA Y EXPLORACIÓN EN LA INSUFICIENCIA RENAL AGUDA: PUNTOS GENERALES

¿Es esto una insuficiencia renal aguda o crónica?

¿Hay otras pistas de la historia?

¿Es probable una causa prerrenal?

¿Podría ser esto una obstrucción?

¿Es probable una enfermedad renal intrínseca?

Fuente: (Brezis \& Rosen, 2015).

\section{¿Es esto una insuficiencia renal aguda o crónica?}

Los datos de laboratorio anteriores, la información de las notas de casos o el médico de cabecera pueden proporcionar la respuesta, pero en muchos casos dicha información no estará disponible. La anemia, la hipocalcemia y la hiperfosfatemia no son buenos indicadores de cronicidad.

La ecografía renal puede mostrar riñones pequeños $(<9 \mathrm{~cm})$, a menudo con cicatrices corticales y posiblemente formación de quistes, en cuyo caso la enfermedad crónica es probable. Lo contrario no siempre es cierto: la presencia de dos riñones de tamaño normal (9 a $14 \mathrm{~cm}$ ) no siempre indica una enfermedad aguda (Glynne, Allen, \& Pusey, 2015).

\section{¿Hay otras pistas de la historia?}

A menudo será necesario un historial específico adicional. ¿Ha tenido una infección de garganta reciente (posible glomerulonefritis posestreptocócica) y, de ser así, un curso de amoxicilina (posible NIA)?. "Un historial farmacológico detallado es importante y debe incluir medicamentos actuales y recientes, y también preparaciones de venta libre (especialmente AINE), drogas recreativas y remedios alternativos o herbales" (Rossert \& Fischer, 2013). El sarpullido, la fiebre y la artralgia también son indicativos de NIA. El dolor de huesos es una característica del mieloma.

\section{Los síntomas constitucionales pueden apuntar a vasculitis sistémica}

Estos con frecuencia preceden a la presentación aguda por muchos meses, que han sido descartados tanto por los pacientes como por los médicos (por ejemplo, congestión nasal y epistaxis como los síntomas iniciales de la granulomatosis de Wegener) y solo salen a la luz durante una revisión detallada de la historia. La hemoptisis podría mostrar un síndrome pulmonar-renal (Rossert \& Fischer, 2013).

En pacientes actualmente o recientemente hospitalizados, los agentes nefrotóxicos como los aminoglucósidos y los medios de radiocontraste deben excluirse rigurosamente. "Después de la angiografía, la función renal tiende a alcanzar su punto más bajo unos días después de la dosis de contraste. Los émbolos de colesterol pueden ocurrir semanas o meses después" (Rossert \& Fischer, 2013).

\section{¿Es probable una causa prerrenal?}

La evaluación del estado hemodinámico del paciente es una parte esencial de la evaluación inicial y la corrección de la hipovo- 
lemia y la hipotensión puede formar parte del tratamiento inicial que salva la vida. "La hipotensión suele ser fácil de detectar, pero puede ser relativa. Una presión sistólica de $110 \mathrm{~mm} \mathrm{Hg}$ en el paciente hipertenso cuyo valor normal es alrededor de $160 \mathrm{~mm} \mathrm{Hg}$ puede comprometer la perfusión renal" (Firth, 2008).

La hipotensión postural (una disminución de la presión arterial sistólica de 20 a 30 $\mathrm{mm} \mathrm{Hg}$ desde la posición acostada a la vertical) y la presión venosa yugular (JVP), medida con el paciente a $45^{\circ}$ con respecto a la horizontal, son marcadores invaluables del estado del volumen. Una disminución postural significativa con la JVP no visible muestra una disminución de volumen y la necesidad de reposición de líquidos, que se lleva a cabo mejor mediante infusiones rápidas y repetidas de pequeños volúmenes $(250 \mathrm{ml})$, a través de un acceso venoso seguro con reevaluación clínica periódica Glynne, Allen, \& Pusey, (2015).

Una vez que el paciente se considera euvolémico, "se debe interrumpir el reemplazo o puede ocurrir edema pulmonar. Si la evaluación de líquidos es difícil, puede ser necesaria la colocación guiada por ultrasonido de un catéter yugular interno para medir la presión venosa central" (Firth, 2008). El procedimiento no está exento de riesgos. Estos se agravan en pacientes con IRA que pueden tener venas colapsadas y un mayor riesgo de hemorragia debido a la coagulopatía y la disfunción plaquetaria.

El tiempo necesario para realizar la tarea no debe retrasar la reanimación con líquidos. La evidencia de una depleción de volumen actual, o una historia que sugiera una reciente, implica una causa prerrenal o NTA, y la respuesta al tratamiento diferencia entre las dos.

\section{¿Podría ser esto una obstrucción?}

La IRA suele ser reversible con un alivio rápido de la obstrucción, pero cuanto más prolongada es la demora, mayor es el daño a largo plazo.

La obstrucción debe buscarse mediante la anamnesis y el examen, antes de proceder a una ecografía urgente del tracto urinario. La enfermedad prostática es una causa común de IRA en los hombres. Se pueden producir síntomas urinarios obstructivos, se puede encontrar una vejiga palpable o próstata hipertrofiada en el examen y / o el cateterismo uretral puede producir un volumen residual significativo. En las mujeres, el carcinoma de cuello uterino puede detectarse en el examen vaginal. Puede haber antecedentes de litiasis renal. La obstrucción puede ocurrir más proximalmente (Thadhani, Pascual, \& Bonventre, 2006).

La ecografía es una excelente herramienta para detectar obstrucciones; no invasivo, relativamente simple, rápido de realizar y portátil. También proporciona información sobre el tamaño renal.

Sin embargo, no es perfecto y no mostrará hidronefrosis en aproximadamente el 5\% de los casos. El recubrimiento de la pelvis renal y los uréteres por tejido maligno o fibrosis retroperitoneal puede resultar en una falla en la dilatación incluso cuando está obstruido, y es posible que no se observe dilatación si hay un elemento prerrenal adicional (con TFG reducida), inmediatamente después de la obstrucción aguda (24 horas), o si la pelvis renal es anormalmente pequeña (una variante anatómica) (Finfer, Bellomo, \& Boyce, 2014).

El diagnóstico debe tenerse en cuenta en aquellos pacientes en los que la obstrucción parece probable sobre la base del cuadro clínico: tomografía computarizada.

\section{¿Es probable una enfermedad renal in- trínseca?}

Se necesita excluir la inflamación renal que 
requiere un diagnóstico y tratamiento urgente para evitar una mayor pérdida y ayudar a la recuperación de la función renal. "La anamnesis y el examen pueden proporcionar pistas (por ejemplo, erupción vasculítica). El análisis de orina es importante y debe realizarse en todos los pacientes que presenten IRA. La hematuria o la proteinuria sugieren una nefropatía intrínseca" (Brezis \& Rosen, 2015). La orina debe ser microscópica y cultivada. Los cilindros de eritrocitos son muy sugestivos de glomerulonefritis, aunque solo están presentes en el 30\% de los casos. Si se considera probable una enfermedad renal intrínseca, es esencial realizar una investigación urgente adicional, al igual que la derivación urgente a un nefrólogo; véanse las secciones posteriores.

\section{Gestión práctica}

El manejo está dirigido a tratar cualquier característica potencialmente mortal, intentar detener o revertir el deterioro de la función renal y, si no tiene éxito, brindar apoyo mediante reemplazo renal anticipando la recuperación renal. La hiperpotasemia, el edema pulmonar y la acidosis grave requieren atención inmediata. El equilibrio de líquidos, el tratamiento de la acidosis menos grave, el uso de diuréticos y dopamina, así como el alivio de la obstrucción, son temas en el tratamiento posterior del paciente, algunos más controvertidos que otros. Siempre que el paciente pueda mantenerse durante el período de inactividad y no se produzcan más agresiones, el riñón es notable en su capacidad para recuperar su función homeostática normal.

\section{Hiperpotasemia}

La hiperpotasemia grave (potasio plasmático $([\mathrm{K}+] \mathrm{p})>6,5 \mathrm{mmol} / \mathrm{l})$ es una emergencia médica debido al riesgo de arritmias cardíacas potencialmente mortales.

A medida que aumenta $[K+] p$, se produce un patrón típico de cambios en el ECG: on- das T en pico / en tienda ( $[\mathrm{K}+] \mathrm{p}>6,5 \mathrm{mmol}$ / I); aplanamiento de la onda P y prolongación del complejo QRS ([K +] p 7-8 mmol / I); ondas sinusoidales ([K+] p 8-9 mmol / I); y fibrilación ventricular o asistolia $([\mathrm{K}+]$ p $>$ $9 \mathrm{mmol} /$ l) (Ahee \& Crowe, 2010).

Sin embargo, estos cambios no siempre son consistentes y los pacientes con un ECG basal normal también pueden desarrollar arritmias. Se debe iniciar un tratamiento urgente de la hiperpotasemia si el potasio sérico es $>6,5 \mathrm{mmol} / \mathrm{l}$, o si hay algún cambio en el ECG. "El tratamiento puede requerir disminución de la ingesta de potasio, modificación de los fármacos que consume el paciente, administración de una resina de intercambio de cationes $y$, en situaciones de emergencia administrar gluconato de calcio, insulina y diálisis" (James \& Lewis, 2020).

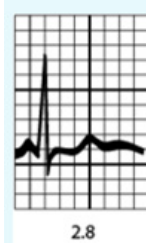

2.8

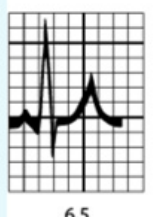

6.5

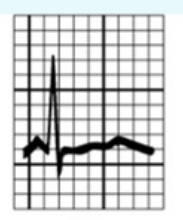

2.5 Hipopotasemia

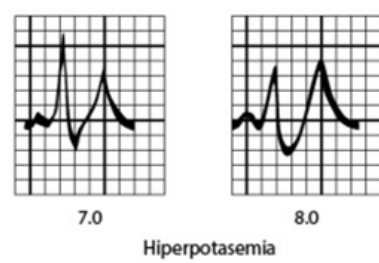

Hiperpotasemia

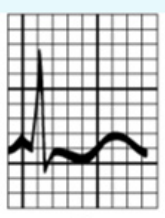

2.0

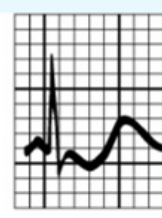

1.7

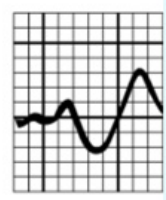

9.0
Figura 1. Patrones electrocardiograficos en la hiperpotasemia.

Fuente: (James \& Lewis, 2020)

\section{Edema pulmonar}

El paciente oligoanúrico con edema pulmonar resultante de una sobrecarga de líquidos (con o sin enfermedad cardíaca subyacente) representa un desafío clínico. "Si existe una falla ventilatoria significativa, ésta debe ser tratada en primer lugar, mediante oxígeno suplementario, ventilación no inva-

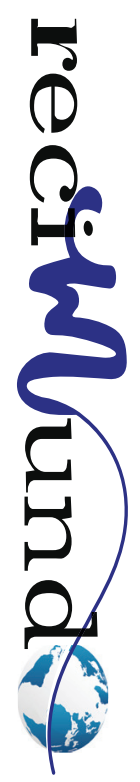


siva o intubación y ventilación, según el estado del paciente" (Brezis \& Rosen, 2015).

Mientras se toman estas medidas, se puede iniciar un tratamiento farmacológico para descargar el corazón descompensado: opioides intravenosos (diamorfina 2,5 a $5 \mathrm{mg}$, con cuidado según el grado de dificultad respiratoria) y una infusión intravenosa de nitrato (por ejemplo, trinitrato de glicerilo) $50 \mathrm{mg}$ en $50 \mathrm{ml}$ de solución salina al 0,9\%, a una velocidad de 2 a $20 \mathrm{ml} / \mathrm{h}$ manteniendo la presión arterial sistólica (95 $\mathrm{mm} \mathrm{Hg}$ ), e intentos realizados para provocar una diuresis.

Se requieren dosis mucho mayores de diuréticos en la insuficiencia renal, por ejemplo, "250 mg de furosemida en $50 \mathrm{ml}$ de solución salina al 0,9\% durante una hora, con un efecto que se observa en una o dos horas, si es que se produce, y que puede repetirse si es eficaz" (Thadhani, Pascual, \& Bonventre, 2006). La venesección es otra medida desesperada.

Si estas intervenciones no tienen éxito, o si el paciente se encuentra en situaciones extremas que parece poco probable que resulten efectivas, la eliminación de líquidos mediante terapia de reemplazo renal es la respuesta definitiva. Se puede usar hemodiálisis o hemofiltración, la elección de la modalidad a generalmente determinada por las necesidades adicionales del paciente, en particular la necesidad de soporte cardiovascular y respiratorio, y el lugar en el que se pueden atender mejor.

El edema pulmonar casi siempre es el resultado de una reanimación excesiva con líquidos, y puede anticiparse en muchos pacientes, especialmente aquellos con disfunción cardíaca conocida, ancianos y aquellos que parecen estar repletos de volumen al principio, y es de esperar que se evite mediante una terapia con líquidos intravenosos más juiciosa.

\section{Acidosis}

La acidosis metabólica grave (pH sanguíneo, 7,2) acompaña a la IRA y surge a través de una variedad de mecanismos, relacionados tanto con la función renal reducida como con la causa subyacente de la enfermedad del paciente. La acidosis sistémica altera la contractilidad cardíaca, induce bradicardia, produce vasodilatación y aumenta la hiperpotasemia, entre otros efectos. Revertir la acidosis mediante la administración de una solución alcalina (bicarbonato de sodio) parece sensato, pero hay muy poca evidencia que demuestre que proporcione beneficios. La terapia en bolo con bicarbonato de sodio hipertónico (8.4\%) "puede empeorar la acidosis intracelular (mientras engaña al médico al mejorar el pH sanguíneo, acidosis paradójica) y causar vasodilatación osmótica, alcalosis metabólica excesiva, hipernatremia y lesión por extravasación si no se administra a una vena central" (Kraut \& Kurtz, 2011).

Las soluciones al $26 \%$ proporcionan una carga de sodio, que provoca la expansión del volumen de líquido extracelular y puede empujar a un paciente que ya está sobrecargado de volumen hacia un edema pulmonar. Las soluciones isotónicas (1,26\%) pueden tener un papel como terapia de reemplazo de líquidos en pacientes estables con una acidosis moderada a severa y un requerimiento de reemplazo de líquidos, en quienes la diálisis no es inminente (Kraut \& Kurtz, 2011)

Por lo general, se requerirá hemodiálisis o hemofiltración para tratar la acidosis grave en pacientes oligoanúricos.

\section{Abordaje clínico}

\section{Equilibrio de fluidos}

Aún se debate cuál es el líquido de reanimación óptimo. 
La solución salina normal y la albúmina al 4\% tienen equivalencia clínica, demostrada por el reciente estudio SAFE de pacientes ingresados en unidades de cuidados intensivos en Australia y Nueva Zelanda, y que revierte estudios previos que muestran que el uso de albúmina puede estar asociado con una mayor mortalidad (Finfer, Bellomo, \& Boyce, 2014).

Cuando el paciente se considera euvolémico (normotenso, sin caída postural, JVP y / o presión venosa central (PVC) normal), se debe tener cuidado para evitar la sobrecarga de líquidos e iniciar un régimen de mantenimiento, que tenga en cuenta las pérdidas renales e insensibles, con el objetivo saldo positivo de 500 ml / día (entrada horaria $=$ salida de la hora anterior más 25 $\mathrm{ml}$ ). Esto requiere una observación precisa y el mantenimiento de registros por parte del personal de enfermería.

\section{Dopamina}

Se ha recomendado el uso de dopamina en dosis bajas (1-3 mg / kg / min) para aumentar la perfusión renal en pacientes en estado crítico. Estudios recientes, incluido un gran ensayo controlado aleatorizado, han demostrado que carece de eficacia sobre el resultado renal o la mortalidad general. El uso de dopamina también puede "reducir la perfusión esplácnica, deprimir la respiración, suprimir la liberación y función de la hormona de la pituitaria anterior y empeorar la función renal en pacientes hipovolémicos o normovolémicos. Actualmente no se justifica su uso rutinario en la IRA" (Galley, 2010).

\section{Diuréticos}

Existe una justificación teórica para el uso de diuréticos de asa en la IRA.

La inhibición de la bomba de $\mathrm{Na}+/ \mathrm{K}+/$ $2 \mathrm{Cl} 2$ en la rama ascendente gruesa del asa de Henle, con la consiguiente disminución de la actividad de $\mathrm{Na}+$ / K + 2ATPasa, debería reducir las necesidades de oxígeno de estas células y, por lo tanto, su susceptibilidad al daño isquémico (Shilliday, Quinn, \& Allison, 2007).

Hay escasos datos clínicos que respalden esto, y estudios recientes han correlacionado el uso de diuréticos con un aumento de la mortalidad. Parece razonable usar solo diuréticos "en pacientes adecuadamente reanimados, pero oligúricos, a una dosis adecuada al grado de insuficiencia renal (250 mg de furosemida por vía intravenosa durante una hora es un régimen estándar) y suspender el tratamiento con diuréticos si persiste la oliguria" (Shilliday, Quinn, \& Allison, 2007). Convertir la insuficiencia renal oligúrica en no oligúrica puede ayudar con el manejo de líquidos y electrolitos.

\section{Alivio de la obstrucción}

Es importante aliviar rápidamente la obstrucción del tracto urinario.

La obstrucción del flujo de salida de la vejiga se puede aliviar mediante el paso de un catéter uretral, que se debe considerar en todos los pacientes con IRA para medir con precisión la producción de orina, pero el alivio de la obstrucción del tracto superior puede requerir ya sea anterógrada (nefrostomía percutánea) o retrógrada (cistoscopia y retrógrado). cateterismo ureteral) (Firth, 2008).

El cateterismo uretral se puede realizar de inmediato, pero otras técnicas requieren planificación. Se requiere una estrecha colaboración entre los servicios de nefrología, urología y radiología y, en muchos casos, puede ser necesaria la terapia de reemplazo renal antes de que se pueda lograr el alivio de la obstrucción.

Una diuresis significativa puede complicar el alivio de la obstrucción completa del tracto urinario, a través de mecanismos

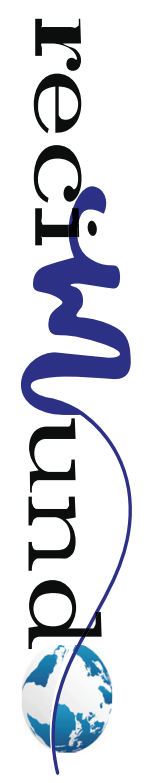


apropiados (excreción de soluto y agua retenidos) e inapropiados (disfunción de la concentración tubular). "La poliuria grave es rara y requiere un tratamiento cuidadoso para prevenir la depleción de volumen y la posible insuficiencia prerrenal, o la reanimación con líquidos excesivamente entusiasta y un impulso adicional a la diuresis" (Glynne, Allen, \& Pusey, 2015).

La obstrucción puede resultar en una respuesta tubular distal alterada a la aldosterona, lo que resulta en una acidosis hipercalémica paradójica cuando aliviado. Por lo general, esto se resuelve de forma espontánea. Un pequeño número tendrá daño tubular permanente y una nefropatía persistente por pérdida de sal.

\section{Conclusiones}

Además de las medidas específicas discutidas anteriormente, la atención continua del paciente con IRA debe estar dirigida a prevenir más agresiones renales y apoyar la fisiología normal. El daño renal adicional generalmente se acumula a través de episodios recurrentes de perfusión renal reducida, a veces difícil de evitar en el paciente inestable, pero también a través de la administración de fármacos nefrotóxicos, con mayor frecuencia AINE, ACE-I y antibióticos aminoglucósidos y glucopéptidos.

Los dos últimos deben dosificarse de acuerdo con las concentraciones plasmáticas controladas. Hay muchos otros fármacos que requieren un ajuste de dosis en función de la TFG (tasa de filtración glomerular) del paciente, no porque sean directamente nefrotóxicos, sino porque la excreción renal alterada cambia su farmacodinamia (por ejemplo, antibióticos cefalosporínicos, morfina). También debe evitarse el uso de medios de contraste.

La IRA se asocia con numerosas alteraciones metabólicas, pero el gasto de energía no aumenta de manera significativa. Sin embargo, las afecciones asociadas, como la sepsis y las quemaduras, conducen a estados hipercatabólicos. Los requerimientos de carbohidratos y proteínas deben adaptarse individualmente e, idealmente, deben administrarse por vía enteral. En algunos casos puede ser necesaria la administración parenteral. Es posible que se requieran vitaminas solubles en agua adicionales, ya que son eliminadas por el TRR (terapia renal de reemplazo).

Existe una mayor susceptibilidad a las infecciones. Las buenas prácticas de control de infecciones y un umbral bajo para considerar una etiología infecciosa de cualquier deterioro clínico pueden minimizar el riesgo. El cuidado de las zonas de presión y la profilaxis contra la trombosis venosa profunda pueden evitar algunos de los problemas de inmovilidad prolongada.

\section{Bibliografía}

Ahee, P., \& Crowe, A. (2010). The management of hyperkalaemia in the emergency department. J Accid Emerg Med, 188-91.

Brezis, M., \& Rosen, S. (2015). Hypoxia of the renal medulla-its implications for disease. $N$ Engl $J$ Med , 647-55.

Feest, T., Round, A., \& Hamad, S. (2003). Incidence of severe acute renal failure in adults: results of a community based study. BMJ , 481-3.

Finfer, S., Bellomo, R., \& Boyce, N. (2014). A comparison of albumin and saline for fluid resuscitation in the intensive care unit. N Engl J Med , 2247-56.

Firth, J. (2008). Medical treatment of acute tubular necrosis. Q J Med , 321-3.

Galley, H. (2010). Renal-dose dopamine: will the message now get through? . Lancet, 212.

Glynne, P., Allen, A., \& Pusey, C. (2015). Acute renal failure in practice. London: Imperial College Press ,156-62.

Hou, S., Bushinsky, D., \& Wish, J. (2003). Hospital-acquired renal insufficiency: a prospective study. Am J Med , 243-8.

James, L., \& Lewis, I. (2020, Abril 19). Brookwood Baptist Health and Saint Vincent's Ascension Health, Birmingham. Retrieved Abril 24, 2021, from Ma- 
nual MSD version para profesionales: https://www. msdmanuals.com/es/professional/trastornos-endocrinol\%C3\%B3gicos-y-metab\%C3\%B3licos/ trastornos-electrol\%C3\%ADticos/hiperpotasemia

Kim, H.-J., \& Han, S.-W. (2012). Therapeutic approach to hyperkalaemia. Nephron , 33-40.

Kraut, J., \& Kurtz, I. (2011). Use of base in the treatment of severe acidaemic states. Am J Kidney Dis ,703-27.

Ortíz, F., \& García, C. (2018). Metodología de la Investigación. México: Vergara.

Rossert, J., \& Fischer, E. (2013). Comprehensive clinical nephrology. 769-7.
Shilliday, I., Quinn, K., \& Allison, M. (2007). Loop diuretics in the management of acute renal failure: a prospective double-blind, placebo-controlled, randomised study. Nephrol Dial Transplant , 2592-6.

Thadhani, R., Pascual, M., \& Bonventre, J. (2006). Acute renal failure. N Engl J Med, 1448-60.

Zambrano, P. (2018). Método Científico. México : Fondo Educativo Interamericano .

\section{CITAR ESTE ARTICULO:}

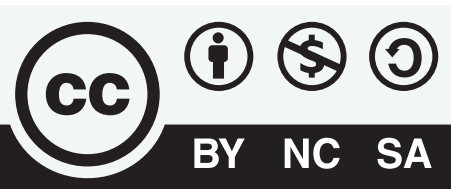

Arguello Molina, T. J., Quirós Rumbea, C. J., Daza Bermeo, K. L., \& Macías Vélez, F. D. (2021). Manejo integral de la insuficiencia renal aguda. RECIMUNDO, 5(2), 128-139. https://doi.org/10.26820/recimundo/5.(2).abril.2021.128-139 